\title{
Modelling and Optimization of Plug Flow Mufflers in Emission Control Systems
}

\author{
Jobin Puthuparampil, Henry Pong \& Pierre Sullivan \\ Version Published \\ Citation Puthuparampil, J., Pong, H., \& Sullivan, P. (2017). Modelling and \\ (published version) Optimization of Plug Flow Mufflers in Emission Control Systems. In \\ SAE 2017 Noise and Vibration Conference and Exbibition (pp. 2017-01- \\ 1782). Grand Rapids, MI: SAE Technical Paper.
}

Additional publisher The final version of this article is available from SAE International information http://doi.org/10.4271/2017-01-1782

How to cite TSpace items

Always cite the published version, so the author(s) will receive recognition through services that track citation counts, e.g. Scopus. If you need to cite the page number of the author manuscript from TSpace because you cannot access the published version, then cite the TSpace version in addition to the published version using the permanent URI (handle) found on the record page.

This article was made openly accessible by $U$ of $T$ Faculty. Please tell us how this access benefits you. Your story matters. 
This document is SAE-copyrighted intellectual property. It may not be shared, downloaded, duplicated, reprinted, or transmitted in any manner without prior written permission from SAE. SAE requires that you make best efforts to secure and protect the document from disclosure, taking at least the same care that you would for your own confidential information.

INTERNATIONAL $\circledast$ Thank you.

\section{Modelling and Optimization of Plug Flow Mufflers in Emission Control Systems}

Jobin Puthuparampil

University of Toronto

\section{Henry Pong}

Safety Power Inc.

\section{Pierre Sullivan}

University of Toronto

CITATION: Puthuparampil, J., Pong, H., and Sullivan, P., "Modelling and Optimization of Plug Flow Mufflers in Emission Control Systems," SAE Technical Paper 2017-01-1782, 2017, doi:10.4271/2017-01-1782.

Copyright (C) 2017 SAE International and Copyright (C) 2017 Institute of Noise Control Engineering

\begin{abstract}
Large-scale emergency or off-grid power generation is typically achieved through diesel or natural gas generators. To meet governmental emission requirements, emission control systems (ECS) are required. In operation, effective control over the generator's acoustic emission is also necessary, and can be accomplished within the ECS system. Plug flow mufflers are commonly used, as they provide a sufficient level of noise attenuation in a compact structure. The key design parameter is the transmission loss of the muffler, as this dictates the level of attenuation at a given frequency. This work implements an analytically decoupled solution, using multiple perforate impedance models, through the transfer matrix method (TMM) to predict the transmission loss based on the muffler geometry. An equivalent finite element model is implemented for numerical simulation. The analytical results and numerical results are then evaluated against experimental data from literature. The transmission loss required in each application of the ECS system will vary depending on the noise profile of the generator in question; therefore, it is necessary to have an effective method of redesigning the muffler to meet the design requirements. Prior work on TMM-based muffler shape optimization utilized complex algorithms such as neural networks and simulated annealing. The present study simplifies the process by using the bounded, limited-memory implementation of the Broyden-Fletcher-Goldfarb-Shanno (BFGS) algorithm in a multi-start framework for shape optimization to achieve the desired transmission loss. By constraining the multi-start method with appropriate design limits, the algorithm is initialized at multiple random points within the design space, ensuring that the solution approaches the global optimum when using a sufficiently large number of initializations.
\end{abstract}

\section{Introduction}

With large-scale (500kW and above) diesel and natural gas generators producing upwards of $120 \mathrm{~dB}$ at the engine exhaust [1], there is a need for acoustic control. Acoustic emission reduction is generally achieved within an emission control system (ECS), which has the primary purpose of reducing the concentrations of combustion byproducts in the exhaust gas stream. Noise reduction in an ECS system can come from the combination of two technologies: dissipative silencing from the porous media required for the chemical scrubbing processes and reactive silencing from a dedicated muffler system. This paper focuses on the modelling and simulation of mufflers used in ECS applications and shape optimization to tune muffler performance to a desired transmission loss characteristic.

Plug flow mufflers provide noise attenuation in a form factor similar to other components in an ECS, such as a diesel particulate filter. This allows for modularized ECS design, streamlining its construction and maintenance. In a plug flow muffler, gas flow enters the first section of the inner tube, is forced out through the perforations on the inner tube to the surrounding annular chamber due to the presence of the flow plug, rejoins in the second portion of the inner tube after passing through the second perforated surface, and finally exits the muffler (Figure 1). The analytical model of a muffler requires the calculation of a $2 \times 2$ transfer matrix, whose elements are sometimes called the "four-pole parameters". This one-dimensional analysis is aptly named the transfer matrix method (TMM).

Sullivan and Crocker proposed the first mathematical model of a perforated muffler and analyzed a non-plug concentric tube muffler [2]. They began by deriving the continuity and momentum equations for the internal tube and external annulus, and then proceeded to solve it in segments using an infinite series. Quick convergence was shown, using only the first 5 terms of the infinite series. Jayaraman and Yam modified Sullivan and Crocker's model with a decoupled solution [ $\underline{3}]$, 
[4] applied to the two crossflow elements found in a plug flow muffler: a perforated expansion and perforated contraction (Figure 2 and Figure 3, respectively). The key improvement was that each muffler element only required one set of calculations, eliminating the need for an infinite series. However, a limitation in their derivation necessitated that the mean flow velocity in the inner tube and the outer annulus had to be the same. This is a non-physical situation as the change in cross-sectional area from the inner to the outer section entails a change in mean flow velocity, except for the zero mean flow case.

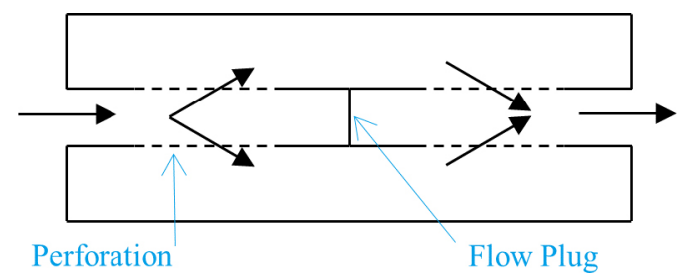

Figure 1. Flow pattern in a plug flow muffler (cross-section)

Munjal et al. addressed this limitation and produced an analytical decoupling method which accounted for the difference in mean flow velocity in the interior tube and exterior annulus [ㄷ]. This was successfully applied to more complex muffler geometries such as the three-duct perforated muffler. Peat addressed the slight numerical instabilities that occurred at peaks in the transmission loss curve for these complex mufflers at non-zero mean flow through the numerical decoupling scheme []].

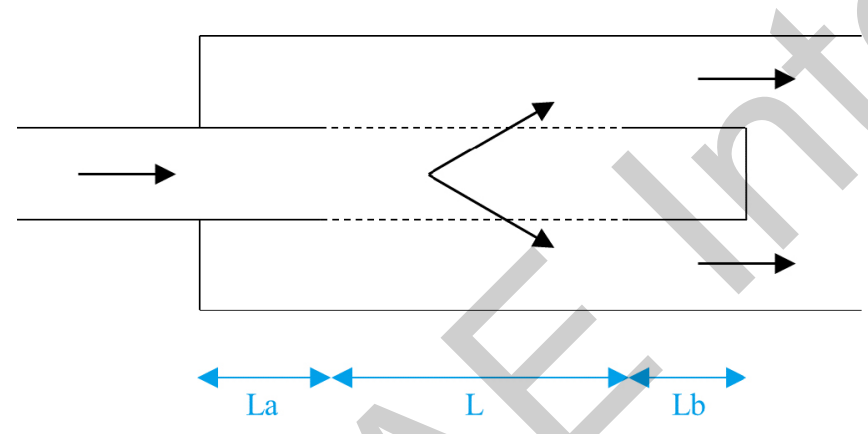

Figure 2. Schematic of a perforated expansion chamber. Image based on [7]

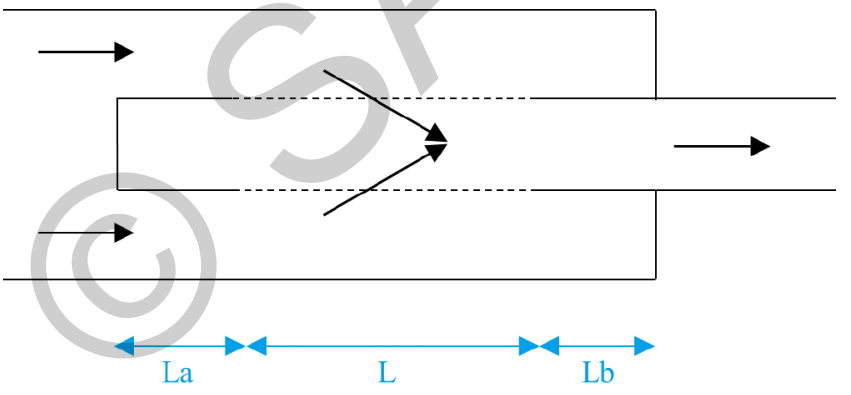

Figure 3. Schematic of a perforated contraction chamber. Image based on [7]

The use of these analytical models in shape optimization was initiated by Yeh et al. on single chamber expansion mufflers [] $]$ and on multi-chamber expansion mufflers [9]. Shape optimization of perforated mufflers was first conducted by Chiu and Chang [10] using algorithms such as simulated annealing [11]-[13], neural networks [14], and genetic algorithms [15]. In most cases, Chiu and Chang's work deals with mufflers used to attenuate air compressors and fans. However, the present work addresses mufflers for internal combustion engines and conducts the optimization through less complex means.
This paper provides a brief overview of Munjal et al.'s analytical transmission loss model, followed by the theory behind the multivariate and multi-start optimization algorithm used. The succeeding section outlines the research methodology, starting with a general overview of the work, and then describes the details of the transmission loss optimization and computational model. The results from the baseline analytical and computational evaluation are compared and discussed. Subsequently, the optimized muffler results are presented and the optimization process is analyzed.

\section{Theory}

\section{Transfer Matrix Method}

The present work utilizes the TMM suggested by Munjal et al. [5] . This method is well suited for the standard plug flow muffler as defined in Figure 1. Unlike typical automotive mufflers, ECS mufflers are larger in diameter with relatively low flow rates, leading to low gas velocities where the Mach number rarely exceeds 0.05 .

Therefore, the present work only considers the zero mean flow case.

TMM supposes that a muffler can be split into a sequence of its sub-components, which can all be analyzed individually, and then combined to reveal their total effect. For a given frequency, each sub-component results in a transfer matrix $T$, such that

$$
\left(\begin{array}{c}
p_{1} \\
\rho_{o} c_{o} u_{1}
\end{array}\right)=T\left(\begin{array}{c}
p_{2} \\
\rho_{o} c_{o} u_{2}
\end{array}\right)=\left[\begin{array}{cc}
T_{A} & T_{B} \\
T_{C} & T_{D}
\end{array}\right]\left(\begin{array}{c}
p_{2} \\
\rho_{o} c_{o} u_{2}
\end{array}\right)
$$

The transfer matrix relates the pressure $(p)$ and the velocity $(u)$ at the inlet of the sub-component, subscript 1 , to the outlet condition, subscript 2. The muffler's complete transfer matrix is

$$
T_{\text {muffler }}=T_{1} T_{2} \ldots T_{n}=\left[\begin{array}{ll}
T_{A} & T_{B} \\
T_{C} & T_{D}
\end{array}\right]_{1}\left[\begin{array}{ll}
T_{A} & T_{B} \\
T_{C} & T_{D}
\end{array}\right]_{2} \ldots\left[\begin{array}{ll}
T_{A} & T_{B} \\
T_{C} & T_{D}
\end{array}\right]_{n}
$$

where the subscript represents the sub-component under consideration. In the specific case of a plug flow muffler, two acoustic sub-components need to be considered $(n=2)$ : a perforated expansion chamber (Figure 2) and a perforated contraction chamber (Figure 3). Munjal details the calculation of the transfer matrices for these sub-components []].

The existence of perforations complicates the transfer matrix calculation as all reliable equations for the perforate impedance $(\xi)$ are experimentally derived. For cross-flow elements where the entire flow must pass through the perforation, the impedance when there is no mean flow is given by Sullivan as [16]

$$
\xi=\frac{p}{\rho_{o} c_{o} u}=\frac{6 * 10^{-3}}{\sigma}+\frac{i k\left(t+0.75 d_{h}\right)}{\sigma}
$$

Bento Coelho provided an alternative formulation [1]

$$
\xi=\frac{p}{\rho_{o} c_{o} u}=\frac{1}{\rho_{o} c_{o}}[R+i X]
$$




$$
\begin{array}{r}
R=\frac{1}{\sigma}\left[\rho_{o}\left(\frac{d^{\prime}}{d_{h}}\right) \sqrt{8 v_{o} \omega}+\left(\frac{\rho_{o}}{8 c_{o}}\right)\left(\omega d_{h}\right)^{2}\right] \\
X=\left(\frac{\omega \rho_{o}}{\sigma}\right)\left(d^{\prime \prime}+\left(\frac{d^{\prime}}{d_{h}}\right) \sqrt{\frac{8 v_{o}}{\omega}}\right) \\
d^{\prime}=t+d_{h} \quad d^{\prime \prime}=t+\left(\frac{8}{3 \pi}\right) d_{h}(1-0.7 \sqrt{\sigma})
\end{array}
$$

Another model for perforate impedance is presented by Bauer [18]

$$
\xi=\frac{p}{\rho_{o} c_{o} u}=\frac{\sqrt{\frac{8 v_{o} k}{c_{o}}\left(1+\frac{t}{d_{h}}\right)}}{\sigma}+\frac{i k\left(t+0.25 d_{h}\right)}{\sigma}
$$

In Equations 3 , $\underline{4}$, and $\underline{5}$, the variables are defined as follows: $k$ is the angular wave number, $t$ is the thickness of the perforated section, $d_{h}$ is the diameter of the perforated holes, $\sigma$ is the area porosity of the perforated sections, $\rho_{o}$ is the ambient air density, $c_{o}$ is the speed of sound in ambient conditions, $v_{o}$ is the ambient kinematic viscosity, and $\omega$ is the angular frequency. These three impedance formulas are explored and compared in this work to determine their accuracies as they relate to modelling plug flow mufflers.

Munjal et al. defined transmission loss (TL) as [ㅁ]

$$
T L=10 \log _{10}\left[\frac{A_{i}}{A_{o}}\left(\frac{1+M_{i n}}{1+M_{a n}}\right)^{2}\left|\frac{T_{A}+T_{B}+T_{C}+T_{D}}{2}\right|^{2}\right]
$$

where $A_{i}$ is the cross-sectional area of the input, $A_{o}$ is the crosssectional area of the output, $M_{i n}$ is the mean flow Mach number within the inner chamber, and $M_{a n}$ is the mean flow Mach number in the outer annulus. $T_{A}, T_{B}, T_{C}, T_{D}$ are the elements of $\mathrm{T}_{\text {muffer }}$ from Equation 2. As this work only considers mufflers with constant inlet and outlet diameters with no mean flow, Equation 6 can be simplified to

$$
T L=10 \log _{10}\left[\left|\frac{T_{A}+T_{B}+T_{C}+T_{D}}{2}\right|^{2}\right]
$$

Since the TMM analysis only considers the propagation of plane waves, it is only fully valid below the cutoff frequency $\left(f_{c}\right)$ for the first circumferential mode [19]; in a circular expansion chamber muffler with zero mean flow [20]],

$$
f_{c}=\frac{1.84 c_{o}}{\pi D_{2}}
$$

where $D_{2}$ is the outer diameter of the muffler. Frequencies higher than $f_{c}$ may have higher order vibrational modes which cannot be captured by TMM.

\section{Optimization -Multi-Variate \& Multi-Start Method}

The constrained multi-variate non-linear optimization process in this problem utilizes the limited-memory BFGS bounded (L-BFGS-B) algorithm. BFGS is a quasi-Newton method that estimates the inverse of the Hessian matrix to guide itself toward a minima. The Limitedmemory BFGS (L-BFGS) decreases the computational cost of the original BFGS algorithm by only storing a subset of the data needed to compute the inverse Hessian matrix. This modification significantly reduces the memory requirements to a linear relationship [21]. A further extension to the L-BFGS algorithm results in the bounded version, L-BFGS-B, which deals with a constrained search space.

It is important to note that L-BFGS-B does not guarantee a global minimum, but a local one. To increase the probability of discovering the global minima, a common technique is to employ a multi-start technique, where multiple optimizations are run in parallel, with each starting at a different initial condition [22] $-[\underline{25}]$. This work only implements a simple version of the multi-start technique, without including clustering.

\section{Muffler Transmission Loss - Finite Element Simulation}

An alternate solution method to predict the TL of a muffler relies on the finite element (FE) numerical discretization of the Helmholtz equation. This equation can be written with pressure as the scalar of interest as

$$
\nabla \cdot\left[-\frac{1}{\rho}\left(\nabla p-\mathbf{q}_{d}\right)\right]-\frac{\omega^{2} p}{\rho c^{2}}=\boldsymbol{Q}_{m}
$$

where $\rho$ is the density, $c$ is the speed of sound, $\omega$ is the angular frequency, $\boldsymbol{Q}_{m}$ is the monopole source term, and $\mathbf{q}_{d}$ is the dipole source term, and the pressure $p$ is a time-harmonic wave of the form

$$
p(\boldsymbol{x}, t)=p(\boldsymbol{x}) e^{i \omega t}
$$

The solid surfaces of the muffler are modelled using hard wall boundary conditions. Plane wave conditions are imposed on the inlet and outlet surfaces, with the inlet surface producing a pressure wave of the desired frequency. The complicated geometry of the perforated section is simplified by applying a constant transfer impedance boundary condition over the perforated section. As with the TMM, the perforation impedance is specified by Equation $3, \underline{4}$, and $\underline{5}$ to discover the differences among them.

Equation 8 can be solved parametrically for a range of frequencies to determine the pressure distribution within a domain. Following this, the transmission loss is calculated using

$$
T L=10 \log _{10}\left(\frac{W_{\text {in }}}{W_{\text {out }}}\right)
$$

where $W_{\text {in }}$ and $W_{\text {out }}$ correspond to the acoustic power at the muffler inlet and outlet, respectively. Acoustic power is calculated using the pressure field solution integrated over the surface $(S)$ of interest (cross-sectional surface of the inlet or outlet)

$$
W_{S}=2 \int_{S} \frac{|p|^{2}}{2 \rho c}
$$




\section{Methodology}

The first step was an evaluation of the baseline muffler used in the ECS system. The muffler was modelled analytically and using FE simulations to determine agreement. The analytical model was then incorporated into an optimization loop so that the muffler geometry could be altered to optimize the transmission loss behavior at desired frequencies. Finally, the solution was modelled through FE simulations to check agreement with the analytical model.

\section{Baseline Model Evaluation}

To assess the validity and accuracy of the proposed modelling techniques, the plug flow muffler geometry presented by Wu et al. was replicated using TMM and FE simulations [26]; the dimensional variables are shown in Figure 4. The results were evaluated using the experimental data presented by $\mathrm{Wu}$ et al. The best performing TMM impedance model (of the three shown in Equations $3, \underline{4}$, and $\underline{5}$ ) was then used in the optimization process.

\section{Transmission Loss Optimization}

\section{Objective Function Definition}

The transmission loss objective function (TLO) used in the optimization process was

$$
T L O=\sum_{i}\left|E S L_{i}-T L_{i}\right| w_{i}
$$

for

$$
i=\{63,125,250,500,1000,2000,4000\}
$$

where $i$ denotes the set of octave band center frequencies of interest, $E S L_{i}$ is the excess sound level at a given octave band, $T L_{i}$ is the averaged muffler transmission loss over the octave band under consideration, and $w_{i}$ is a weight applied to each octave band that determines the band's contribution to the TLO.

The excess sound level $\left(E S L_{i}\right)$ is defined as

$$
E S L_{i}=E N_{i}-N C_{i}
$$$$
\text { ( }
$$

where $E N_{i}$ is the measured engine noise reported in decibels and $N C_{i}$ is the Noise Criterion standard required for the application [27]. The difference between the two quantities naturally provides a suitable TL target for the design. The present work uses NC-60 as the desired noise criterion; the maximum permissible output noise level for each octave band is provided in Table 1 along with the measured sound emitted by the engine $\left(E N_{i}\right)$ (CAT C27 $750 \mathrm{~kW}$ generator at 1 meter). Together, Equations 12 and 13 provide a metric that is correlated to the radiated noise excess beyond $\mathrm{NC}-60$ limits, without directly considering the muffler's termination reflection.

The averaged muffler transmission loss over an octave band $\left(T L_{i}\right)$ is found as

$$
T L_{i}=10 \log _{10}\left(\sum_{j=\text { Lower Limit }, i}^{\text {Upper Limit }, i} 10^{(0.1)\left(T L_{j}\right)} / n_{i}\right)
$$

where $n_{i}$ is the number of elements summed for a given octave band. The upper and lower frequency limits for a given octave band is listed in Table 1. Note that the frequency limits are rounded to the nearest $10 \mathrm{~Hz}$, as the solution is calculated with a $10 \mathrm{~Hz}$ resolution over the frequency range from $10 \mathrm{~Hz}$ to $4000 \mathrm{~Hz}$.

Table 1. Octave Band Range and Associated Engine Noise, Noise Criterion, and TLO Weights

\begin{tabular}{|c|c|c|c|c|c|}
\hline $\begin{array}{c}\text { Octave } \\
\text { Band } \\
(\mathbf{H z})\end{array}$ & $\begin{array}{c}\text { Lower } \\
\text { Limit } \\
(\mathbf{H z})\end{array}$ & $\begin{array}{c}\text { Upper } \\
\text { Limit } \\
(\mathbf{H z})\end{array}$ & $\begin{array}{c}\text { Engine } \\
\text { Noise }(\boldsymbol{E} \boldsymbol{N}) \\
(\mathbf{d B})\end{array}$ & $\begin{array}{c}\text { NC-60 } \\
(\mathbf{d B})\end{array}$ & $\begin{array}{c}\text { TLO } \\
\text { Weight } \\
\left(\boldsymbol{w}_{\boldsymbol{i}}\right)\end{array}$ \\
\hline 63 & 40 & 90 & 98.3 & 77 & 0.2 \\
\hline 125 & 90 & 180 & 107.1 & 71 & 0.2 \\
\hline 250 & 180 & 360 & 102.5 & 67 & 0.2 \\
\hline 500 & 360 & 710 & 102.5 & 63 & 0.1875 \\
\hline 1000 & 710 & 1420 & 104 & 61 & 0.1525 \\
\hline 2000 & 1420 & 2840 & 100.8 & 59 & 0.055 \\
\hline 4000 & 2840 & 4000 & 96.5 & 58 & 0.005 \\
\hline
\end{tabular}

The TLO weights $\left(w_{i}\right)$ in $\underline{\text { Table } 1}$ were chosen (a) to prioritize the frequency ranges where a muffler is traditionally used as an effective method of noise attenuation and (b) to account for the inherent inaccuracies that occur above the cutoff frequency. Mufflers, or reactive silencers in general, show excellent performance at low frequency while dissipative silencing is typically used to address higher frequencies [28]. In an ECS, dissipative acoustic noise reduction occurs as a secondary function of the porous media used for the chemical processes. Therefore, it is beneficial to focus the muffler's silencing towards lower frequencies where dissipative silencing is not very effective. The second reason for the choice of weights is to ensure that the more accurate low-frequency portion of the prediction has more contribution to Equation 12 than the highfrequency range, which has errors due to the plane wave assumption of the TMM. However, overall TL predicted in the high-frequency range is useful, leading to non-zero weights in these octave bands.

\section{Optimization Implementation}

The Munjal et al. TMM for a plug flow muffler [7] was implemented in Python 2.7 utilizing the numpy module for matrix operations. The L-BFGS-B algorithm from the scipy.optimize module was executed in a parallel fashion using the $p p$ module for an efficient randomized multi-start method. It minimized Equation 12 by changing the physical parameters defining the shape of the muffler. Table 2 shows the complete list of dimensional parameters and their bounds. The limits are informed by physical constraints and manufacturing constraints. Both sections of the inner perforated tube are defined as having equal dimensions to simplify the manufacturing process. The variables $D_{2}, t_{1}, t_{2}, d h_{1}$, and $d_{2}$ were held constant as they were seen to have a comparatively low effect on the TL in a sensitivity study. Therefore, these variables are set to their respective values from the Wu et al. muffler. 
The optimization was performed 2000 times in parallel on an 8-core computer. Each initialization of the optimization algorithm begins at random values between the limits prescribed in Table 2 , striving towards a minima, terminating when

$$
\frac{T L O_{k-1}-T L O_{k}}{\max \left(T L O_{k}, T L O_{k-1}, 1.0\right)}<1 \cdot 10^{-4}
$$

where TLO from Equation 12 evaluated at the current iteration $k$ and the previous iteration $k-1$.

Table 2. Shape Optimization Bounds and Constraints

\begin{tabular}{|c|c|c|c|}
\hline Parameter & Description & Low & High \\
\hline $\mathbf{D}_{\mathbf{2}}$ & Outer diameter & \multicolumn{2}{|c|}{$0.1076 \mathrm{~m}$} \\
\hline $\mathbf{r D}$ & $\begin{array}{c}\text { Ratio of inner and outer } \\
\text { diameter }\end{array}$ & 0.4 & 0.8 \\
\hline $\mathbf{L} \mathbf{a}_{\mathbf{1}}=\mathbf{L a}_{2}$ & $\begin{array}{c}\text { Length of } 1^{\text {st }} \text { solid } \\
\text { section }\end{array}$ & $0.0 \mathrm{~m}$ & $0.15 \mathrm{~m}$ \\
\hline $\mathbf{L}_{1}=\mathbf{L}_{\mathbf{2}}$ & $\begin{array}{c}\text { Length of perforated } \\
\text { section }\end{array}$ & $0.05 \mathrm{~m}$ & $0.25 \mathrm{~m}$ \\
\hline $\mathbf{L b}_{1}=\mathbf{L b}_{\mathbf{2}}$ & $\begin{array}{c}\text { Length of 2 } \\
\text { section }\end{array}$ & $0.0 \mathrm{~m}$ & $0.15 \mathrm{~m}$ \\
\hline $\boldsymbol{\sigma}_{\mathbf{1}}=\boldsymbol{\sigma}_{\mathbf{2}}$ & Perforation porosity & $14 \%$ & $25 \%$ \\
\hline $\mathbf{t}_{\mathbf{1}}=\mathbf{t}_{\mathbf{2}}$ & Thickness of inner tube & \multicolumn{2}{c|}{$0.0011938 \mathrm{~m}$} \\
\hline $\mathbf{d h}_{1}=\mathbf{d h}_{\mathbf{2}}$ & Perforation diameter & \multicolumn{2}{|c|}{$0.00635 \mathrm{~m}$} \\
\hline
\end{tabular}

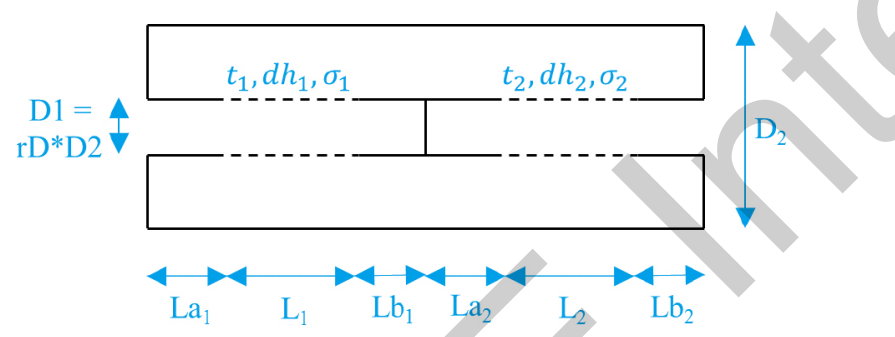

Figure 4. Muffler schematic showing dimensions used in shape optimization

\section{Finite Element Simulation Setup}

The FE simulation was conducted through COMSOL Multiphysics 5.2a using the Acoustics Module with a parametric frequency sweep study. The mufflers were implemented with both two-dimensional axisymmetric (2DA) and three-dimensional (3D) geometry. First, the geometry of the muffler was parametrically modelled. Plane wave boundary conditions were applied at the inlet and outlet, along with hard wall boundary conditions at all relevant surfaces. The perforated boundaries were defined via custom functions representing Equations $\underline{3}, \underline{4}$, and $\underline{5}$. A free triangular mesh (for 2DA) and a tetrahedral mesh (for 3D) was used, ensuring that the maximum element size was limited by

$$
\text { Max.Size }=\frac{c_{o}}{10 f_{\max }}
$$

where $f_{\max }$ was the maximum frequency of interest. Equation 16 ensures that the model always has enough spatial resolution to resolve the smallest wavelength under consideration [29]. TL solutions were obtained for a parametric frequency sweep from $10 \mathrm{~Hz}$ to $4000 \mathrm{~Hz}$ with a resolution of $10 \mathrm{~Hz}$.

\section{Results \& Discussion}

\section{TMM \& FE Model Evaluation}

The first step was an evaluation of the TMM and FE models by comparison against experiments conducted by Wu et al [26]. The TL results from the TMM are presented in Figure 5 alongside the experimental data. The experimental data up to $3200 \mathrm{~Hz}$ shows three major TL peaks occurring at $747 \mathrm{~Hz}, 1770 \mathrm{~Hz}$, and $2062 \mathrm{~Hz}$ [26]. The Sullivan and Bento Coelho models are in qualitative agreement with the experimental data up to roughly $2200 \mathrm{~Hz}$. Substantial deviations only begin after the cutoff frequency, $f_{c}=1877 \mathrm{~Hz}$, and are associated with the plane wave assumption inherent to the TMM. However, both the Sullivan and Bento Coelho models predict the general decrease in TL above $2200 \mathrm{~Hz}$. The Bauer model, while containing the 3 major peaks, exhibits an increasing frequency shift at higher frequencies; the first peak is shifted by $42 \mathrm{~Hz}$, the second peak by $220 \mathrm{~Hz}$, and the third peak by $270 \mathrm{~Hz}$. This behavior is currently unexplained. Of the three impedance models used in the TMM, the Sullivan model performs best, as corroborated by the high Pearson correlation coefficient listed in Table 3 .

Table 3. Pearson Correlation Coefficient (Prediction vs. Experimental Data)

\begin{tabular}{|c|c|c|c|}
\hline Impedance Model & TMM & 2DA & 3D \\
\hline Sullivan & 0.8084 & 0.6683 & 0.6681 \\
\hline Bento Coelho & 0.7445 & 0.7880 & 0.7878 \\
\hline Bauer & 0.2696 & 0.8496 & 0.8495 \\
\hline
\end{tabular}

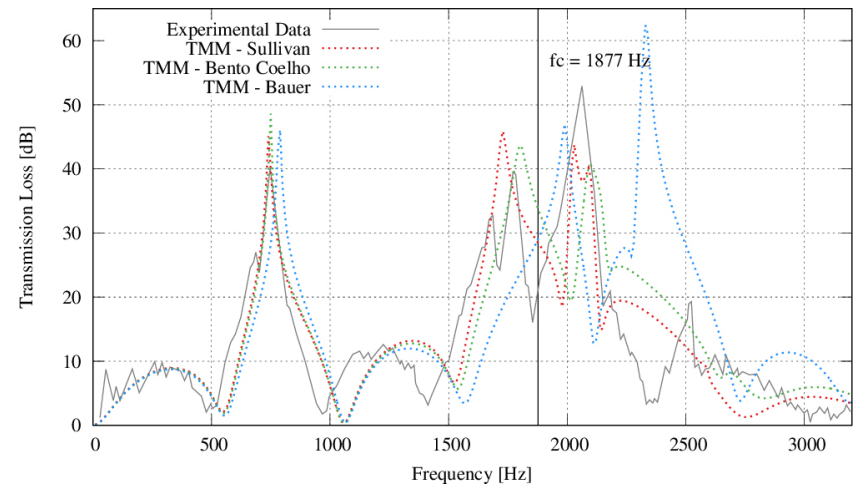

Figure 5. TMM evaluation - transmission loss

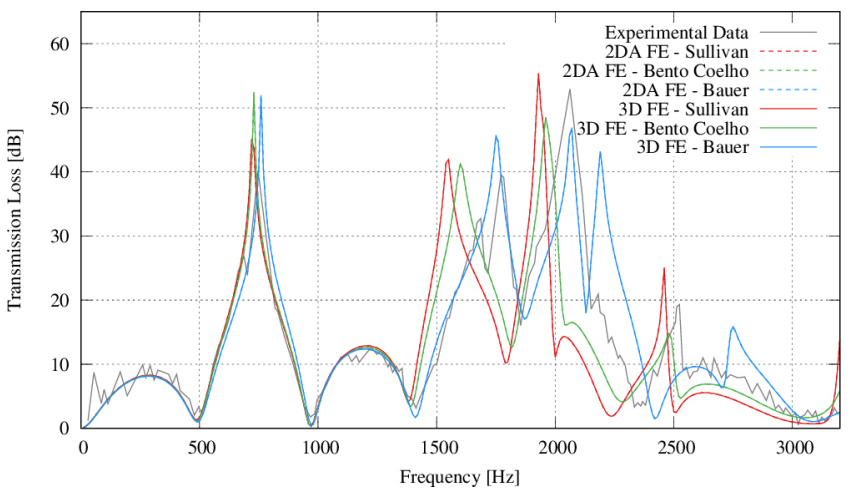

Figure 6. FE evaluation - transmission loss

Both 2DA and 3D FE simulation results are shown in Figure 6. The 3D simulations took 70 times the computational time when compared to the 2DA model, primarily because of the 100 times increase in the number of elements. The 2DA results lie almost coincident with the 
$3 \mathrm{D}$ results for the range of frequencies considered, and it is achieved at a significantly lower computational cost. This implies that there are no 3D modes of pressure distribution until $3200 \mathrm{~Hz}$.

Of the three impedance models used in the FE simulations, the Bauer model shows the highest level of qualitative accuracy, contrary to the results from the TMM. These findings are further verified by the high correlation coefficient for the FE Bauer model seen in Table 3. Both the Sullivan and Bento Coelho models show significant deviation from approximately $1400 \mathrm{~Hz}$ onwards. However, certain similarities exist across TMM and FE - the order in which each impedance model peaks stays consistent. For example, when considering the second peak in the experimental data $(1770 \mathrm{~Hz})$, the Sullivan model peaks first, followed by the Bento Coelho, and finally the Bauer model. This trend is seen for all TL peaks in both TMM and FE.

The observations in this section lead to adopting the Sullivan impedance model for TMM and the Bauer model for 2DA FE simulations, as they provide the highest correlation and qualitative agreement with experimental data for their respective modelling methods.

\section{Transmission Loss Optimization}

\section{Optimized Solution}

The optimization process was conducted to design a muffler that reduced the noise output of a generator to the NC-60 standard. 2000 random initializations of the optimization were conducted in 5 hours and 40 minutes with only 3 instances of the optimizer failing. The dimensions of the best performing design along with its objective function value is given in Table 4. Figure 7 shows the associated TMM and FE transmission loss predictions. Based on the results from the model evaluation section, the FE Bauer model is expected to predict the actual TL with a much higher accuracy than the TMM Sullivan model. Thus, the current section compares the TMM results against FE simulations.

Table 4. Optimized Muffler - Dimensions

\begin{tabular}{|c|c|}
\hline Variable & Value \\
\hline $\mathrm{rD}$ & 0.4000 \\
\hline $\mathrm{La}_{1}=\mathrm{La}_{2}$ & $0.1498 \mathrm{~m}$ \\
\hline $\mathrm{L}_{1}=\mathrm{L}_{2}$ & $0.1144 \mathrm{~m}$ \\
\hline $\mathrm{Lb}_{1}=\mathrm{Lb}_{2}$ & $0.06003 \mathrm{~m}$ \\
\hline$\sigma_{1}=\sigma_{2}$ & $18.24 \%$ \\
\hline Objective Function (TLO) & $12.86 \mathrm{~dB}$ \\
\hline
\end{tabular}

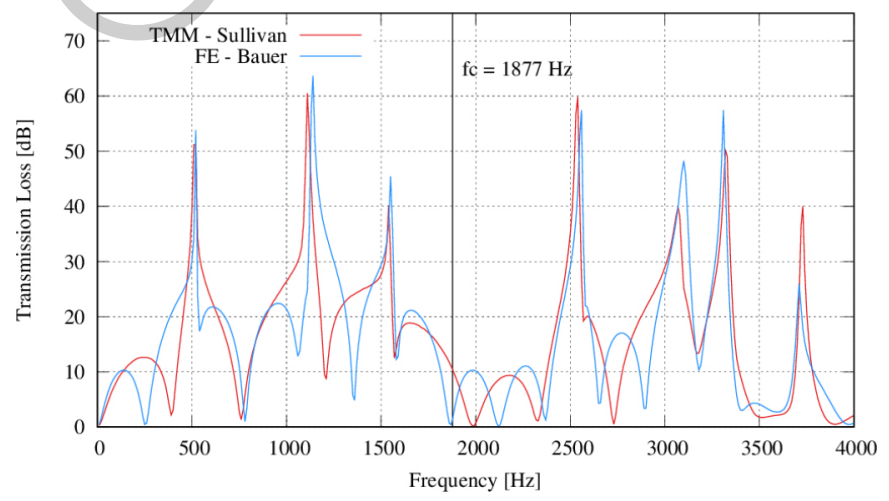

Figure 7. Optimized muffler - transmission loss
All 7 major TL peaks, especially the 4 up to $2550 \mathrm{~Hz}$, are predicted equally well by both the TMM and FE. Additionally, general TL levels agree across both modelling methods, with a region of 3 peaks up to approximately $1570 \mathrm{~Hz}$, after which there is relatively low TL to $2400 \mathrm{~Hz}$, following which 4 peaks occur. Th accuracy is reflected in the high correlation coefficient of 0.7520 between the two prediction methods.

Figure 8 presents the final noise output from the engine and muffler system based on the TMM and FE predictions for the optimized muffler. The output noise sound pressure level (SPL) is defined as

$$
S P L_{i}=E N_{i}-T L_{i}
$$

and both the TMM and FE results match extremely well. This is because the Noise Criterion standard only considers an average over an octave band calculated through Equation 14, disregarding small range frequency mismatch.

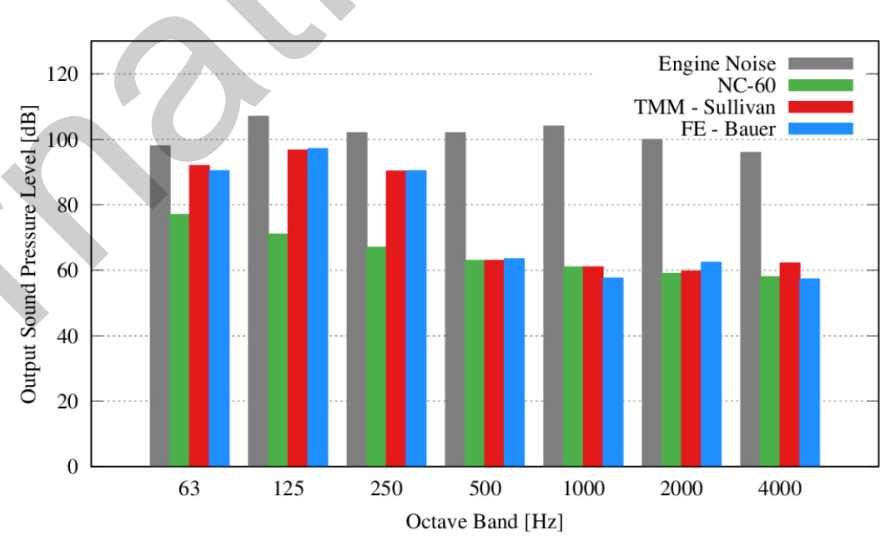

Figure 8. Original engine noise, NC-60 criterion, and the output sound pressure levels of the optimized muffler

However, a large discrepancy is observed between the acceptable output SPL determined by NC-60 and the optimized muffler system's output SPL in the $63 \mathrm{~Hz}, 125 \mathrm{~Hz}$, and $250 \mathrm{~Hz}$ octave bands (the low-frequency range). In fact, this difference is the major contributor to the non-zero evaluation of the objective function seen in Table 4. The inability for the optimized muffler to meet the low-frequency targets is related to the $\mathrm{rD}$ variable and its chosen lower limit of 0.4 ; TMM exhibits prediction inaccuracies below this value. However, a strong inverse proportionality between $\mathrm{rD}$ and low-frequency muffler TL was found with an FE parametric study where $\mathrm{rD}$ was varied from 0.1 to 0.8 , keeping all other parameters constant at their values from Table 4. Figure 9 shows the output SPL for the parametric study; a muffler with $\mathrm{rD}=0.1$ easily outperforms the NC-60 criterion. However, choosing such a small diameter for the inner tube introduces manufacturing challenges, lack of structural integrity if porosity is held constant, and increased pressure drop if porosity is reduced to maintain structural integrity [을. 


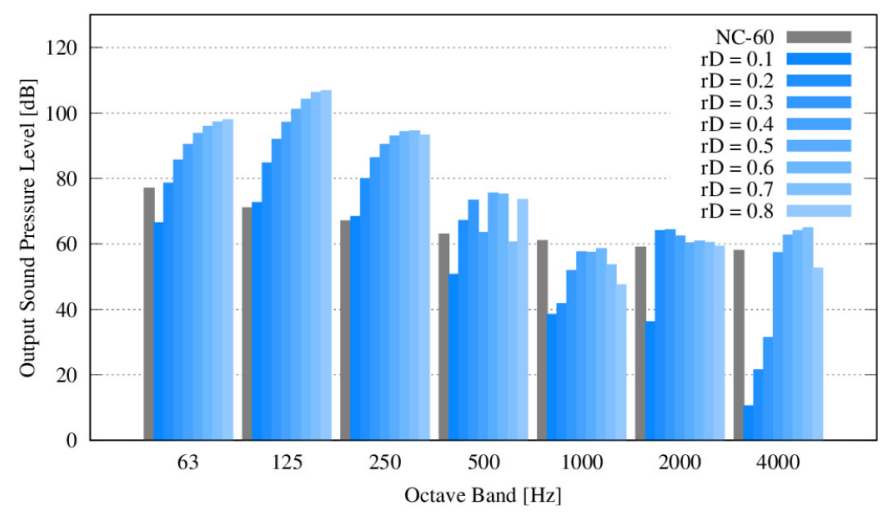

Figure 9. Parametric study - output sound pressure levels (SPL)

\section{Optimization Analysis}

A histogram of TLO evaluations of the final result of all 1997 successful optimizations is seen in Figure 10. It reveals that only $1.8 \%$ of optimizations reached the lowest recorded bin, while $45.1 \%$ of optimizations terminated between TLO $=(17,19.5]$. Thus, the use of a multi-start algorithm is justified and necessary to find the best performing local minima.

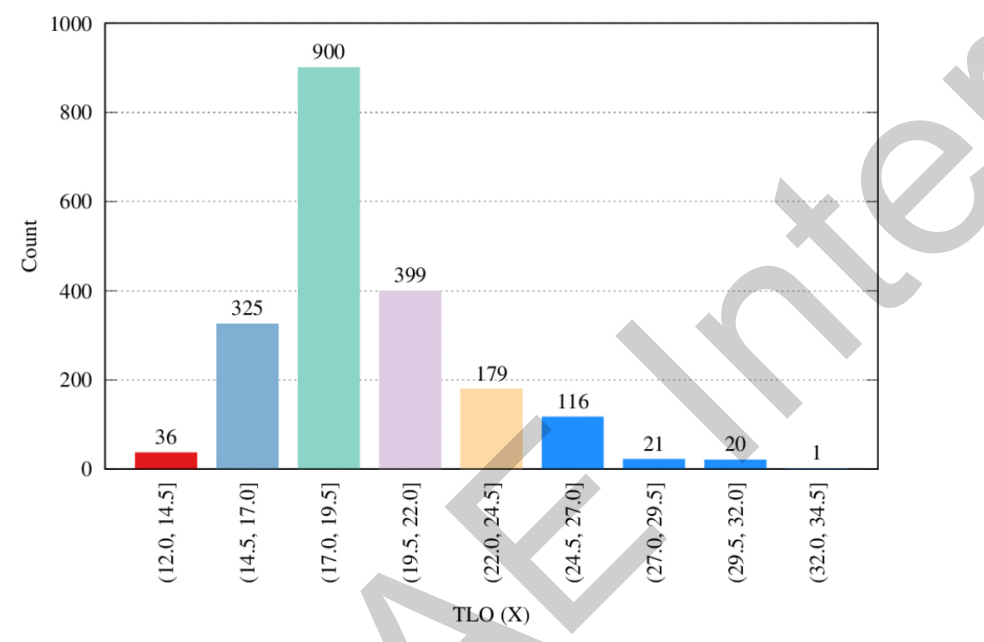

Figure 10. Histogram of TLO evaluations at the final result of each optimization

Of the 1997 solutions discovered, 1756 of them were unique local minimas. This 5-dimensional solution space can be visualized through dimensionality reduction. Principal component analysis (PCA) was conducted on a z-score standardized dataset of the unique solutions to map the space onto 2 independent variables which maximized the variance of the projection [31]. Due to the correlation between the $\mathrm{La}$ and $\mathrm{Lb}$ values, two major clusters with very similar behavior are seen. Thus, focusing on one of these clusters in the PCA provides a clearer visualization of underlying trends, as shown in Figure 11. A region of well-performing solutions is observed surrounding the point $(0,-1)$, as marked by the circle labelled 'Optimal Solution Cluster'. The majority of the low-TLO solutions lie within this region, including the previously discussed bestperforming design.

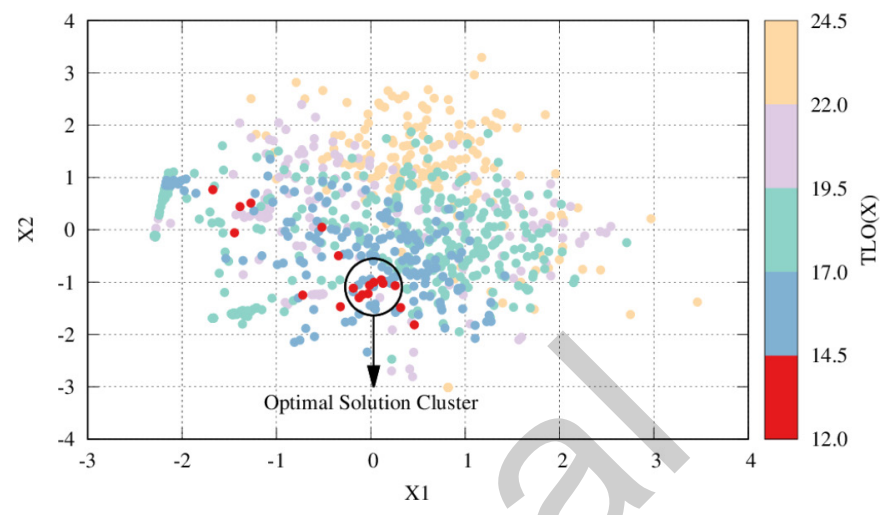

Figure 11. Dimension-reduced visualization of one cluster of 883 unique solutions (local minimas), colored by their associated TLO evaluation

Identifying a region of significance such as this provides an effective method to check whether a given set of muffler dimensions will perform well, simply based on the X1 and X2 values it maps on to. This check can be executed using the PCA transformation coefficients on the z-score standardized muffler dimension values, given as

$$
\begin{gathered}
X 1=\left[\begin{array}{c}
0.6440 \\
-0.08345 \\
-0.3862 \\
0.6166 \\
-0.2213
\end{array}\right] \cdot\left[\begin{array}{c}
z-\operatorname{score}(r D) \\
z-\operatorname{score}(L a) \\
z-\operatorname{score}(L) \\
z-\operatorname{score}(L b) \\
z-\operatorname{score}(\sigma)
\end{array}\right] \\
X 2=\left[\begin{array}{c}
0.3263 \\
-0.6727 \\
-0.1524 \\
-0.3273 \\
0.5573
\end{array}\right] \cdot\left[\begin{array}{c}
z-\operatorname{score}(r D) \\
z-\operatorname{score}(L a) \\
z-\operatorname{score}(L) \\
z-\operatorname{score}(L b) \\
z-\operatorname{score}(\sigma)
\end{array}\right]
\end{gathered}
$$

\section{Conclusion}

In conclusion, this work investigated the modelling and optimization of plug flow mufflers used in large scale generator emission control systems. Firstly, experimental results from a baseline muffler model was used to evaluate the accuracy of the TMM and FE simulations using 3 different perforate impedance models. TMM and FE performed best using the Sullivan and Bauer models respectively. The TMM model was then incorporated into a multi-start optimization process using the L-BFGS-B algorithm. The use of a relatively simple algorithm provides the solution at a low computational cost, as opposed to previous work in the field which used complex methods such as neural networks and genetic algorithms. The optimization aimed to reduce the noise output of a generator to the levels permitted by the NC-60 Noise Criterion standard. The optimizer was successful in designing a muffler that handled the noise from the $500 \mathrm{~Hz}$ to 4000 $\mathrm{Hz}$ octave bands. However, the low-frequency range $(63 \mathrm{~Hz}$ to $250 \mathrm{~Hz}$ octave bands) was not adequately silenced. This was due to conservative limits placed on the $\mathrm{rD}$ parameter to avoid inaccuracies in the TMM modelling.

A general analysis of the results generated by each initialization of the optimizer revealed the importance of the multi-start algorithm used in this problem. As the problem has a highly non-linear solution space with 1756 local minimas discovered in the given bounds, there is a high probability that any individual initialization of the optimizer 
will perform poorly. A region of optimal solutions in the solution space was identified around the point $(0,-1)$ through dimensionality reduction and visualization. Additionally, an explicit relationship was provided to check if a given set of muffler dimensions would fall within the optimal region, allowing for quick and computationally efficient redesign during the muffler development stage.

\section{References}

1. Aaberg D., "Generator set noise solutions :Controlling unwanted noise from on-site power systems," 2007.

2. Sullivan J. W. and Crocker M. J., "Analysis of concentric-tube resonators having unpartitioned cavities," J. Acoust. Soc. Am., vol. 64 , no. 1, p. 207, 1978.

3. Jayaraman K. and Yam K., "Decoupling approach to modeling perforated tube muffler components," J. Acoust. Soc. Am., vol. 69, no. 2, p. 390, 1981.

4. Thawani P. T. and Jayaraman K., "Modeling and applications of straight-through resonators," J. Acoust. Soc. Am., vol. 73, no. 4, pp. 1387-11389, 1983.

5. Munjal M. L., Rao K. N., and Sahasrabudhe A. D., "Aeroacoustic analysis of perforated muffler components," $J$. Sound Vib., vol. 114, no. 2, pp. 173-188, 1987.

6. Peat K. S., "A numerical decoupling analysis of perforated pipe silencer elements," J. Sound Vib., vol. 123, no. 2, pp. 199-212, 1988

7. Munjal M. L., "Muffler Acoustics," in Formulas of Acoustics, 2nd ed., Mechel F. P., Ed. New York: Springer, 2008.

8. Yeh L. J., Chang Y. C., Chiu M. C., and Lay G. J., "Computeraided optimal design of a single-chamber muffler with side inlet/outlet under space constraints," J. Mar. Sci. Technol., vol. 11, no. 4, pp. 189-196, 2003.

9. Yeh L.-J., Chang Y.-C., and Chiu M.-C., "Application of genetic algorithm to the shape optimization of a constrained doublechamber muffler with extended tubes," J. Mar. Sci. Technol., vol. 12, no. 3, 2004.

10. Chiu M. C. and Chang Y. C., "Shape optimization of multichamber cross-flow mufflers by SA optimization," J. Sound Vib., vol. 312, no. 3, pp. 526-550, 2008.

11. Chang Y. C. and Chiu M. C., "Shape optimization of onechamber perforated plug/non-plug mufflers by simulated annealing method," Int. J. Numer. Methods Eng., vol. 74, no. 10, pp. 1592-1620, 2008.

12. Chang Y.-C. and Chiu M.-C., "The optimal design of multichamber side mufflers equipped with perforated cross-flow tubes and intruding tubes using simulated annealing," J. Mech., vol. 27, no. 3, 2011.

13. Chiu M.-C. and Chang Y.-C., "Shaped optimization of multichamber mufflers with open-ended perforated inlets using a simulated annealing method," in 24th National Conference on Noise Control Engineering 2010, Noise-Con 10, Held Jointly with the 159th Meeting of the Acoustical Society of America, 2010, vol. 3 .

14. Chang Y.-C., Chiu M.-C., and Cheng M.-M., "Optimum design of perforated plug mufflers using a neural network and a genetic algorithm," Proc. Inst. Mech. Eng. Part C J. Mech. Eng. Sci., vol. 223, no. 4, pp. 935-952, 2009.
15. Chiu M.-C. and Chang Y.-C., "Shape optimization of multichamber side inlet/outlet mufflers hybridized with multiple perforated intruding tubes using a genetic algorithm," J. Mar. Sci. Technol., vol. 21, no. 3, 2013.

16. Sullivan J. W., "A method for modeling perforated tube muffler components. II. Applications,” J. Acoust. Soc. Am., vol. 66, no. 3, p. 779,1979 .

17. Datchanamourty B., "Detailed Modelling of Mufflers With Perforated Tubes Using Substructure Boundary Element Method," University of Kentucky, 2004.

18. Bauer A. B., "Impedance Theory and Measurements on Porous Acoustic Liners," J. Aircr., vol. 14, no. 8, pp. 720-728, Aug. 1977.

19. Binois R., Dauchez N., Ville J.-M., Perrey-Debain E., and Beillard G., "Modeling of cylindrical baffle mufflers for low frequency sound propagation," in Acoustics, 2012.

20. Eriksson L. J., "Higher order mode effects in circular ducts and expansion chambers," J. Acoust. Soc. Am., vol. 68, no. 2, p. $545,1980$.

21. Byrd R. H., Lu P., Nocedal J., and Zhu C., "A Limited Memory Algorithm for Bound Constrained Optimization," SIAM J. Sci. Comput., vol. 16, no. 5, pp. 1190-1208, Sep. 1995.

22. Dixon L. C. W. and Jha M., "Parallel algorithms for global optimization," J. Optim. Theory Appl., vol. 79, no. 2, pp. 385395, Nov. 1993.

23. Voglis C. and Lagaris I. E., "Towards 'Ideal Multistart'. A stochastic approach for locating the minima of a continuous function inside a bounded domain," Appl. Math. Comput., vol. 213, no. 1, pp. 216-229, 2009.

24. Tu W. and Mayne R. W., "An approach to multi-start clustering for global optimization with non-linear constraints," Int. J. Numer. Methods Eng., vol. 53, no. 9, pp. 2253-2269, Mar. 2002.

25. Boender C. G. E., Rinnooy Kan A. H. G., Timmer G. T., and Stougie L., "A stochastic method for global optimization," Math. Program., vol. 22, no. 1, pp. 125-140, Dec. 1982.

26. Wu T. W., Zhang P., and Cheng C. Y. R., "Boundary Element Analysis of Mufflers With an Improved Method for Deriving the Four-pole Parameters," J. Sound Vib., vol. 217, no. 4, pp. 767779, 1998.

27. Beranek L. L., "Balanced noise - criterion (NCB) curves," $J$. Acoust. Soc. Am., vol. 86, no. 2, pp. 650-664, Aug. 1989.

28. Sound Research Laboratories, Noise Control in Industry, 3rd ed. CRC Press, 2002.

29. "COMSOL - The Acoustics Module User's Guide." 2013.

30. Munjal M. L., Vijayasree N. K., and Paruchuri C., "Flow resistance network analysis of the back-pressure of automotive mufflers," Indian J. Eng. Mater. Sci., vol. 20, no. 5, pp. 336-349, 2013.

31. Ringnér M., "What is principal component analysis?," Nat. Biotechnol., vol. 26, no. 3, 2008.

\section{Contact Information}

Jobin Puthuparampil jobin.puthuparampil@utoronto.ca

5 King's College Road, Toronto, Ontario, Canada, M5S 3G8 


\section{Acknowledgments}

The authors thank Henry Pong, Rahul Jain, and Robert Stelzer from Safety Power Inc. (SPI) for sharing their knowledge and experience with emission control systems and mufflers. The authors also acknowledge MITACS and SPI for providing support, both financially and data, and CMC and SOSCIP for providing access to software and computational resources, respectively.

\section{Definitions/Abbreviations}

2DA - two-dimensional axisymmetric

3D - three-dimensional

BFGS - Broyden - Fletcher - Goldfarb - Shanno

ECS - emission control system

EN - engine noise

ESL - excess sound level

FE - finite element

L-BFGS-B - bounded limited-memory BFGS

NC - Noise Criteria

SPL - sound pressure level

TL - transmission loss

TLO - transmission loss objective

TMM - transfer matrix method $c_{o}$ - ambient speed of sound

$\boldsymbol{d}_{\boldsymbol{h}}$ - perforation diameter

$\boldsymbol{f}$ - frequency

$f_{c}$ - cutoff frequency

$\boldsymbol{i}$ - imaginary unit

$\boldsymbol{k}$ - angular wave number

$\boldsymbol{L}$ - muffler half-length

$\boldsymbol{M}$ - Mach number

$\boldsymbol{v}_{\boldsymbol{o}}$ - ambient kinematic viscosity

$\omega$ - angular frequency

p - pressure

$\boldsymbol{q}_{\boldsymbol{d}}$ - dipole source term

$\boldsymbol{Q}_{\boldsymbol{m}}$ - monopole source term

$\boldsymbol{\rho}_{\boldsymbol{o}}$ - ambient density

$\boldsymbol{\sigma}$ - area porosity of perforation

$\boldsymbol{t}$ - perforated tube thickness

$\boldsymbol{u}$ - velocity

$\boldsymbol{w}_{\boldsymbol{i}}$ - TLO function weight

$\boldsymbol{W}$ - acoustic power

$\boldsymbol{\xi}$-perforation impedance

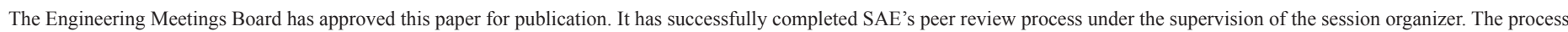
requires a minimum of three (3) reviews by industry experts.

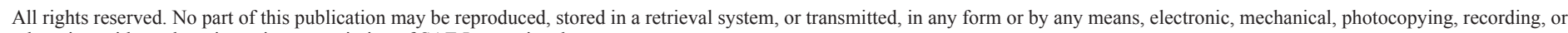
otherwise, without the prior written permission of SAE International.

Positions and opinions advanced in this paper are those of the author(s) and not necessarily those of SAE International. The author is solely responsible for the content of the paper.

ISSN 0148-7191

http://papers.sae.org/2017-01-1782 